Jörg Rehn presented a material of Ioo severe and selected cases with multiple injuries which were treated from 1962 to 1965 . In conclusion we want to compare these cases under two aspects with the material we reviewed ourselves.

I. Incidence of accidents: here, the percentage of accidents at work is higher in paraplegics (Table XI). This is easily explained by the fact that until a few years ago we admitted only professional accidents.

2. Incidence of specific injuries: in both reviews we find an almost even percentage of injuries to the skull. In every other respect, however, the two curves differ widely. Certainly the reason for this discrepancy lies partly in the aspects under which these cases were compiled. The first group was selected solely according to the degree of injury, while the second group comprised only cases with injuries of the spinal cord. We nonetheless thought that a comparison would be worthwhile as it shows distinct differences.

\title{
REFERENCES
}

REHN, JöRG (I966). Probleme der Diagnostik und des schweren Unfalls. Hft. Unfallheilk, 87, II I-II7.

SCHLAG, G. (I967). Abdominelle Katheterparazentese beim stumpfen Bauchtrauma. Chirurgie, 2, 17-20.

\section{ASSOCIATED FRACTURES OF THE LIMBS IN TRAUMATIC PARAPLEGIA AND TETRAPLEGIA}

\author{
By Jean Bénassy, M.D. (Paris) \\ Hopital Poincaré, Garches
}

WE will relate only, and briefly, our cases of traumatic paraplegia and tetraplegia with associated fractures of the paralysed limbs. Amongst our 250 most recent cases 16 patients, with important fractures have been studied ( 2 tetraplegics and I4 paraplegics). In all there were 28 fractures involving femur (I0), hip (5), ankle (5), os calcis and astragalus (8). One of the patients, a paraplegic below $\mathrm{T}_{4}$, with a severe injury to the chest, had 5 associated fractures.

It could appear that the lower the level of the spinal cord lesion the higher the percentage of the fractures of the lower limbs, the site of these becoming correspondingly lower. In fact, the two tetraplegic cases had their fractures high up in the femur, while the cases of lumbar fractures were frequently associated with bilateral fractures of the os calcis.

Prognosis.-On the whole quite good, which is one of the rare optimistic chapters in paraplegia because:

(I) pain being absent an anaesthetic is not necessary.

(2) flaccidity, in the early days, seems to allow easy reduction.

(3) later on, the absence of pain makes results tolerable, which, in particular for the os calcis, would have been quite impossible for normal patients. 
(4) mostly, consolidation occurs with great rapidity, six weeks instead of the usual ten weeks, taking place with a hypertrophic callus similar to an osteoma in juxta-articular fractures.

Nevertheless, this beneficial rate of healing is found only in upper neurone lesions, never in a lower neurone involvement. On the other hand, this phenomena is encountered only in the two upper thirds of the limbs, never in the lower third or in the ankles.

Fractures of the Os Calcis. These are often bilateral, opened, with an increased risk of osteitis. As far as paraplegic patients are concerned the treatment of these fractures can be reduced as the radiological results have little importance, the pain factor being absent.

Fractures of the Legs below the Knee. In all five cases, two of which were open (one of which had to be amputated before arrival at Garches).

Amongst the closed fractures, one had to be treated after six months by a secondary graft, the primary one having failed.

Osteosynthesis can yield good results, but plaster of Paris seems to be an even simpler method, in good and experienced hands, the danger of pressure sores being remote.

On the other hand, one treatment seems dangerous, and should be avoided, namely the uni- or bi-polar extension, which, because of the flaccid lesion would lead to pseudarthrosis as well as render regular turning difficult.

Fractures of the Femur. This chapter is quite different. We have treated nine cases, eight out of which have healed after osteosynthesis, nailing, plating and external fixation. Two of these patients were tetraplegics. One had a ruptured spleen and was operated immediately before the reduction. Another had a ruptured bladder and spleen, another had a total of seven fractures, six of which were in the non-paralysed area. As far as femoral fractures are concerned, plaster of Paris and continuous extension seem to us a regrettable procedure. Osteosynthesis appears to be the best method as it allows the normal treatment of paraplegia as well as ensuring a rapid healing of the fracture. Before deciding on open reduction of a fracture in a paraplegic patient, one must keep in mind the susceptibility of these patients to haemorrhage. This can be demonstrated by two observations:

One is historical, as shown by numerous and precise observations of Ollivier of Angers, France, in 1827. His observations are even the more interesting as they were always nearly followed by a detailed post-mortem report. In that time, following the teaching of Broussais, paraplegics in France as well as probably in England, were treated by abundant blood lettings. Death very often followed such a 'therapeutic' procedure in a matter of days or hours, much quicker than it would have taken place 'normally' in those patients. Recently, we think, we might have found an answer to this problem by studying, in I6 patients, the blood mass, using intravenous radioactive Iodine I3I, fixed to a serum-albumin. The dosage was made possible thanks to the Volomectron electronic apparatus. The results seem to show in paraplegic, but mainly in tetraplegic patients, that the blood volume is increased as compared with the normal subject ( $800 \mathrm{ml}$.). Only paraplegics in a very bad condition, suffering from sores, have a blood mass 
close to normal. One can put forward the hypothesis that the increased blood mass is necessarily connected with an impaired oxygenation of the tissues in a paraplegic as a result of the ortho-sympathetic impairment. It explains too the sensitivity of these patients to bleeding and the need for replacing rigorously the least loss of blood, pre- or post-operatively.

Theoretical Considerations. The most strange phenomenon is the accelerated consolidation of the fractures in upper motor neuron lesions and localised in the proximal two-thirds of the paralysed lower limbs. These are exactly the same circumstances of development of osteomas in the same patients. But this phenomena is far from being the privilege of paraplegics. We can see it happen with great frequency in head injuries where also fractures heal with greatest rapidity, in spite of the difficulties of treatment, even in absence of any treatment at all. Equally we encounter the formation of an hypertrophic callus in two circumstances: in anoxemia developing in complications following general anaesthetic, or, associated with extensive burns, in haemorrhage which can accompany apparently most ordinary injuries.

In all these instances including those of neurogenic origin, the influencing factor is the lack of oxygen supply of the fractured bony substance.

One can demonstrate this phenomenon in the paraplegic patient as his venous blood keeps all the properties of the arterial blood $\mathrm{O}_{2}, \mathrm{CO}_{2}, \mathrm{p} . \mathrm{CO}_{2}, \mathrm{pH}$ and alkaline reserve. We have been able to show that this phenomenon was caused by the crippling of the ortho-sympathetic system. As for the absence of these phenomena at the level of the lower third of the limbs, one can propose the particular distribution of the venous supply at that level of the diaphysis. In a normal bone one can, without exaggerating, say that the blood enters the bone by the diaphysal artery and leaves the bone by the metaphysal veins. Between these two systems exists, similar to that of the liver, a special drainage system where without doubt, the bone-forming proteins on which, secondary, the phospho-calcic salts deposit, is developed. But, the drainage of the distal articulations of the limbs is not the same as the proximal articulations. It is done by superficial veins and not by a deep network. This explains, perhaps, the two different types of consolidation, as the unequal localisation of osteomas.

\title{
TRAUMATIC PARAPLEGIA AND ASSOCIATED FRACTURES
}

\author{
By Drs. A. Tricot and R. Hallot \\ Centre de Traumatologie et de Réadaptation, Hôpital Universitaire Brugmann, \\ Bruxelles 2, Belgium
}

IN 1962 we had the opportunity to give a first report on the same subject.

We had included 270 paraplegic cases for our study. Twenty-eight of these were noted with associated fractures, the proportion giving a percentage of approximately io per cent. (Table I). 